\title{
Surface Impedance Boundary Conditions applied to solving nonlinear electrodynamic systems
}

\author{
Stanislaw Pawlowski, Jolanta Plewako \\ The Faculty of Electrical and Computer Engineering \\ Rzeszow University of Technology \\ Wincentego Pola 2, 35-959 Rzeszów, Poland \\ e-mail: \{spawlo/jplewako\}@prz.edu.pl
}

(Received: 28.03.2017, revised: 05.05.2017)

\begin{abstract}
The paper presents a method for solving electromagnetic field problems by applying impedance boundary conditions for systems including conducting bodies of nonlinear magnetic properties in an excited harmonic field. Three types of impedance boundary conditions were derived. A procedure for computing surface impedance based on the transfer matrix is proposed. This procedure has been tested by comparing computational results to calculations where time function distortions were considered, and to the experimental data.
\end{abstract}

Key words: nonlinear electrodynamics, Surface Impedance Boundary Conditions, harmonic fields, quasi-stationary fields, skin effect

\section{Introduction}

Surface Impedance Boundary Conditions (SIBC) have been successfully applied to solving electrodynamic problems for complex geometry systems (see e.g. [1-7], more extensive references on this subject can be found in [3]). SIBC are to be applied to systems where skin effect occurs. It is related to tangential components of the electric $\boldsymbol{E}$ and magnetic field $\boldsymbol{H}$ on the conducting surface being mutually normal, and their ratio, so called surface impedance, to be constant. Such dependencies allow to split electromagnetic field distribution calculations into separate, unrelated problems that can be formulated separately for each specific area of the system under consideration.

The basic requirements for effective SIBC application include electromagnetic penetration depth to be small in comparison to the curvature radius of the interface, and the field function to be only slightly variable along the tangents to these surfaces. Such conditions are easily satisfied in electromagnetic systems of high frequencies (e.g. radio frequencies). The skin effect is strongly pronounced there. Low frequency systems (e.g. power-line frequencies) may prove fairly compliant and eligible for SIBC application [4-7] - as well. 
Commonly SIBC are developed for systems of linear and homogenous medium that is of constant electromagnetic parameters. Nevertheless, such an assumption does not provide prerequisites for developing SIBC [1]. Wherever analysed systems cover either inhomogeneous, or non-linear areas, it is still necessary to modify SIBC and the methodology for computing specific parameters, as presented in chapter 3 of this paper.

A general method for solving nonlinear, quasi-stationary problems of 3D electrodynamics with SIBC was proposed $[8,9,11]$. It is based on Fundamental Solutions Method (FSM). Magnetic field distribution is found iteratively as self-consistent distribution of the magnetic field at magnetically active surfaces (i.e. ferromagnetic) of magnetic permeability determined from the given magnetisation curve $\mu=\mu(H)$. Introductory numerical experiments performed for model problems confirmed the method to be correct and fairly convergent. However, only a simplified determination of SIBC parameters was assumed, specifically, while developing the formulas an assumption on linearity was made, and only a properly modified, local value for magnetic permeability based on the magnetisation curve were substituted into. Obviously, such an approach disregards e.g. the magnetic permeability dependence on the distance from the conductor surface, which may significantly contribute to errors. Therefore, the next step was to develop a more precise procedure for determining local surface impedance values [11].

The procedure made use of the transfer matrix concept and applied it to compute electromagnetic field distribution in the layer structures [10]. The skin area beneath the conductor surface divided into discreet elements, within which a constant (invariant in time) value, as of the magnetisation curve, for the magnetic permeability is assumed. It means that under a harmonic excitation field, the induced field functions are harmonic as well. Under such an assumption, the analysis of electrodynamic systems is much facilitated. It allows to disregard time dependence by introducing Maxwell equations in their complex form. Still, the problem arises how to determine a substitute magnetic permeability for particular elements of the skin layer. In this paper, the procedure reported elsewhere [11] has been completed by adding two methods for averaging the value of magnetic permeability.

Moreover, a way of considering an approximated magnetic hysteresis by applying a complex value for the magnetic permeability has been proposed [13, 14]. A series of numerical tests have been performed. Results have been compared both to the simulation results where field function distortions due to time dependences were included, and to the experimental results presented elsewhere [14].

\section{Surface Impedance Boundary Conditions}

\subsection{A linear approach}

As it was stated in the Introduction, SIBC are applicable where skin effect occurs, i.e. where the electromagnetic field penetrates not too deeply into the areas located under the conductive surface. It can be assumed that, for cases where such areas are significantly greater in size than the substitute field penetration depth (massive conductor cases), their internal electromagnetic field is limited to a thin layer under the surface, which is called a skin layer. The basic differential operations in such areas are presented in the Appendix. 
By applying these formulas and as long as the assumption that all $s_{1}, s_{2}$ derivatives of the electromagnetic field components are negligible in comparison with their respective $s_{3}$ derivatives holds, a general relationship between the intensities of the electric and magnetic field at the conductor-dielectric boundary surface can be expressed as [1-3]:

$$
\boldsymbol{n} \times \boldsymbol{E}=Z_{c} \boldsymbol{n} \times(\boldsymbol{n} \times \boldsymbol{H}),
$$

where $Z_{c}$ denotes surface impedance, $\boldsymbol{n}$ - stands for the unit vector perpendicular to the interface.

This relationship is called a surface impedance boundary condition (SIBC) and it means that both the electric $\boldsymbol{E}_{t}$ and magnetic $\boldsymbol{H}_{t}$ field components tangent to the boundary surface are mutually normal, and the ratio of their magnitudes:

$$
Z_{c}=\frac{\boldsymbol{E}_{t}}{\boldsymbol{H}_{t}}
$$

depends solely on the local properties of the adjacent areas, i.e. their electromagnetic parameters, and on the field frequency.

In the homogenous media of linear properties the surface impedance can be expressed by the formula [1-3]:

$$
Z_{c}=\sqrt{\frac{\mathrm{j} \omega \mu}{\gamma+\mathrm{j} \omega \varepsilon}},
$$

where: $\mu, \varepsilon, \gamma$ and $\omega$ stand for magnetic permeability, permittivity, electric conductivity, and pulsation, respectively.

It shall be underlined here that neither homogeneity, nor linearity of the electromagnetic parameters of the areas, provides the necessary assumption while applying the condition (1) [3]. Nonetheless, dependence (3) does not hold for nonlinear media and the local $Z_{c}$ magnitude should be then determined numerically. In chapter 3 a numerical procedure for determining $Z_{c}$ for magnetically nonlinear media is provided. It is based on the transfer matrix approach.

Practical arrangements for applying SIBC numerically in a form (1) may prove troublesome, as all the components for $\boldsymbol{E}$ and $\boldsymbol{H}$ fields must be computed simultaneously. Fortunately, other more convenient SIBC formulation can be found, where parameters related to material properties and field frequency other than $Z_{c}$ occur.

Below, three other SIBC representations for which fields need to be harmonic and quasistationary (i.e. for which the Maxwell displacement current is negligible) and conductive areas linear are presented $[2-4,8,11]$ :

$$
\begin{gathered}
\boldsymbol{J}=\alpha \boldsymbol{n} \times \boldsymbol{H}, \\
\frac{\partial H_{n}}{\partial n}=-\beta H_{n}, \\
\overline{\bar{\Delta}} V_{m}=\beta \frac{\partial V_{m}}{\partial n},
\end{gathered}
$$


where: $\boldsymbol{J}$ stands for current density at the interface, $V_{m}$ is the magnetic potential $\left(\boldsymbol{H}=-\operatorname{grad} V_{m}\right)$, $H_{n}$ - normal component of magnetic field, $\overline{\bar{\Delta}}$ - surface Laplacian (see (A10)),

$$
\begin{aligned}
& \alpha=\sqrt{j \omega \gamma \mu}, \\
& \beta=\mu_{0} \sqrt{\frac{j \omega \gamma}{\mu}} .
\end{aligned}
$$

Though the above relationships result from condition(1), stable material parameters are assumed for their derivation. In the chapter that follows analogous dependencies are derived for SIBC in the nonlinear (applicable also to non-homogenous) systems.

\subsection{Condition (4) for nonlinear systems}

Due to $\boldsymbol{E}_{t}$ and $\boldsymbol{H}_{t}$ components being mutually normal, and from (2) it can be concluded that (1) can be rewritten in the form:

$$
\boldsymbol{E}_{t}=Z_{c} \boldsymbol{n} \times \boldsymbol{H} .
$$

Additionally, as the condition on the continuity for the tangent components of both electric and magnetic fields holds, clearly this dependence is correct on both sides of the interface, i.e. on the dielectric and conductor side alike. On the conductor side, i.e. within the skin layer, the normal component of the electric field does not occur [1], hence

$$
\boldsymbol{E}=\boldsymbol{E}_{t} .
$$

With the differential Ohm's law taken into consideration

$$
\boldsymbol{J}=\gamma \boldsymbol{E}
$$

and (20) (9) we arrive at

$$
\boldsymbol{J}=\gamma Z_{c} \boldsymbol{n} \times \boldsymbol{H} .
$$

By comparing (23) (12) to (15) (4) it may be concluded that the condition has taken on the form which is identical to the one for the linear case, as long as

$$
\alpha=\gamma Z_{c} .
$$

\subsection{Condition (5) for nonlinear systems}

Let us assumed that all the field components are sinusoidal functions of time. Though such an assumption does not hold for systems including media of nonlinear properties, the error due to such a simplification can be acceptable in electrodynamics. Comparisons of the computational data performed under such an assumption with computations where time dependent distortions in the time field function were taken into account confirmed this supposition. The conclusion was also supported with comparison of the experimental data [12] presented in chapter 4.

To obtain the sought relationships let us consider a certain neighbourhood of any point $\mathrm{P}$ within the thin layer area including the dielectric - conductor boundary surface (Fig. A1). The 
thickness of this sector is assumed to be equal to $2 \eta$ and to be large enough to include entire skin layer of the conducting area; it means that for $s_{3}>\eta$ the field is negligible. Within the coordinate system $s_{1}, s_{2}, s_{3}$ introduced in the Appendix we have $\boldsymbol{n}=[0,0,1]$. By denoting

$$
\boldsymbol{E}=\left[E_{1}, E_{2}, E_{3}\right], \quad \boldsymbol{H}=\left[H_{1}, H_{2}, H_{3}\right]
$$

and making use of (9)

$$
E_{1}=Z_{c} H_{2}, \quad E_{2}=-Z_{c} H_{1} .
$$

Within the dielectric sector of the concerned area, as based on Faraday's law

$$
\operatorname{rot} E=-\mathrm{j} \omega \mu_{0} H .
$$

By decomposing from (A8) the third component $\operatorname{rot} E$

$$
\frac{1}{h_{1} h_{2}}\left(\frac{\partial}{\partial s_{1}}\left(h_{2} E_{2}\right)-\frac{\partial}{\partial s_{2}}\left(h_{1} E_{1}\right)\right)=-j \omega \mu_{0} H_{3} .
$$

By substituting the dependence (15) to (17), with a few simple operations and applying (A9) and (A11)

$$
Z_{c} \overline{\overline{\operatorname{div}}} \boldsymbol{H}+\boldsymbol{H} \cdot \overline{\overline{\operatorname{grad}}} Z_{c}=\mathbf{j} \omega \mu_{0} H_{3} .
$$

According to (A7) and (A11)

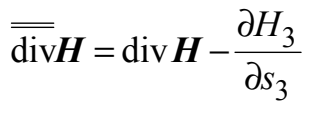

and as $\operatorname{div} \boldsymbol{H}=0$ for the dielectric area, so

$$
\frac{\partial H_{3}}{\partial s_{3}}=-\frac{\mathrm{j} \omega}{Z_{c}} H_{3}+\frac{1}{Z_{c}} \boldsymbol{H} \cdot \overline{\overline{\operatorname{grad}}} Z_{c} .
$$

As $s_{3}$ represents the component normal to the boundary surface, finally

$$
\frac{\partial H_{n}}{\partial n}=-\beta H_{n}+\frac{1}{Z_{c}} \boldsymbol{H} \cdot \overline{\overline{\operatorname{grad}}} Z_{c},
$$

where

$$
\beta=\frac{j \omega \mu_{0}}{Z_{c}} .
$$

Relation (21) is a generalised SIBC (5) under the simplified assumptions for the electromagnetic field to be harmonic and quasi-stationary. 


\subsection{Condition (6) within a nonlinear approach}

For quasi-stationary fields, within the dielectric part of the considered system a scalar magnetic potential can be introduced:

$$
\boldsymbol{H}=-\operatorname{grad} V_{m},
$$

that satisfies Laplace's equation

$$
\Delta V_{m}=0 .
$$

By substituting (23) to (20)

$$
-\frac{\partial^{2} V_{m}}{\partial s_{3}^{2}}=-\beta \frac{\partial V_{m}}{\partial s_{3}}-\frac{1}{Z_{c}} \operatorname{\operatorname {grad}} V_{m} \cdot \overline{\overline{\operatorname{grad}} Z_{c}}
$$

and on the base of (A6), (A10) and (24) observing that

$$
\frac{\partial^{2} V_{m}}{\partial s_{3}^{2}}=-\overline{\bar{\Delta}} V_{m},
$$

we arrive at the sought generalisation of SIBC for the scalar magnetic potential, namely

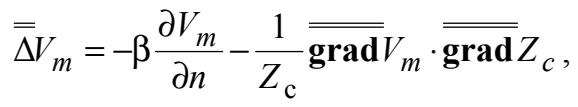

with $\beta$ derived as in formula (22) (33).

\section{Procedure for computing surface impedance}

Applying SIBC formulas (1), (12), (21), and (27) to solving electromagnetic field problems in systems including conducting areas of nonlinear magnetic properties the surface impedance $Z_{c}$ distribution at the boundary surfaces for these areas is required to be known. However, local magnitude of $Z_{c}$ is known to depend on the magnetic permeability, which in turn depends on the magnetic field intensity. Therefore, the procedures for determining $Z_{c}$ and computing field distribution need to be interrelated. A transfer matrix method based concept for numerical procedure to compute surface impedance was presented elsewhere [10,11]. Further in this chapter a brief summary of it, completed with some significant supplements is provided.

Accordingly to the assumptions made earlier, within the conducting sector of the system under consideration the area where electromagnetic field occurs is limited to the skin layer beneath the boundary surface (see Fig. 1). The relation $\mu=\mu(H)$ for this area is assumed to be known. The relationship is assumed to be unique, though $\mu$ can be also a complex value, which allows to incorporate an approximated magnetic hysteresis effect $[12,13]$.

The first step of the proposed procedure is discretization by dividing of the skin layer on the surfaces being parallel and perpendicular to the interface, into small volume elements $\Omega_{i, j, k}$, as shown in Fig. 1. Magnetic permeability within the element is assumed to be constant (the 
distribution of magnetic permeability is approximated by the step function). It shall be noticed here that under the assumption made earlier, the field is variable, and consequently also magnetic permeability variations are much more pronounced along the normal direction than along the directions tangent to the surface, and thus the elements width $d_{k}$, (compare Fig. 1) shall be respectively smaller than their dimensions along the directions $s_{1}, s_{2}$.

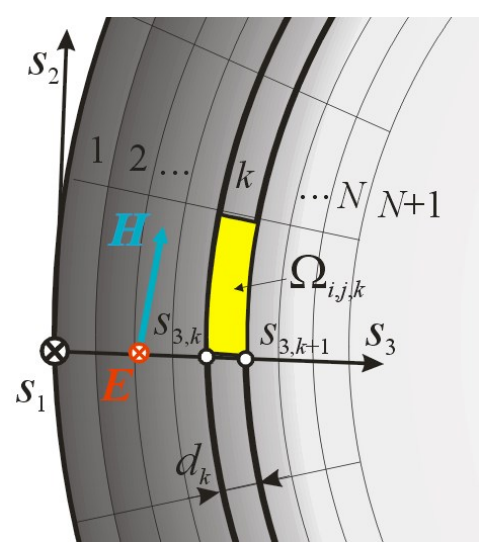

Fig. 1. Skin layer area - illustrated method for computing surface impedance; $i, j, k$ means numbering indices in directions $s_{1}, s_{2}, s_{3}$ (respectively)

With no limitations to the generality of the considerations it can be assumed that within the selected element axis $s_{1}$ is parallel to the vector $\boldsymbol{E}$, thus

$$
\boldsymbol{E}=\left[E_{1}, 0,0\right], \quad \boldsymbol{H}=\left[0, H_{2}, H_{3}\right] .
$$

With such assumptions, the electromagnetic field components in the element $\Omega_{i, j, k}$ (see Fig. 1) can be approximated with the functions listed below (to further simplify the notation, indexes $i, j$ are ignored).

$$
\begin{gathered}
E_{1, k}=E_{1, k}^{+}\left(s_{1}, s_{2}\right) e^{-\alpha_{k}\left(s_{3}-s_{3, k}\right)}+E_{1, k}^{-}\left(s_{1}, s_{2}\right) e^{\alpha_{k}\left(s_{3}-s_{3, k}\right)}, \\
H_{2, k}=H_{2, k}^{+}\left(s_{1}, s_{2}\right) e^{-\alpha_{k}\left(s_{3}-s_{3, k}\right)}+H_{2, k}^{-}\left(s_{1}, s_{2}\right) e^{\alpha_{k}\left(s_{3}-s_{3, k}\right)}, \\
H_{3, k}=H_{3, k}^{+}\left(s_{1}, s_{2}\right) e^{-\alpha_{k}\left(s_{3}-s_{3, k}\right)}+H_{3, k}^{-}\left(s_{1}, s_{2}\right) e^{\alpha_{k}\left(s_{3}-s_{3, k}\right)},
\end{gathered}
$$

where $s_{3, k}$ denotes the initial coordinate of the $k^{\text {th }}$ sector (compare Fig. 1),

$$
\alpha_{k}=\sqrt{\mathrm{j} \omega \gamma \mu_{i, j, k}}, \mu_{k}
$$

is substitute magnetic permeability of $\Omega_{k}$ element. To further simplify the notation, indexes $i, j$ are ignored.

According to Faraday's law the following relations are obtained

$$
\frac{E_{1 k}^{+}}{H_{2 k}^{+}}=-\frac{E_{1 k}^{-}}{H_{2 k}^{-}}=Z_{k}=\sqrt{\frac{\mathrm{j} \omega \mu_{k}}{\gamma}},
$$




$$
\begin{aligned}
& H_{3 k}^{+}=\frac{1}{\mathrm{j} \omega \mu_{k}}\left(\frac{\partial E_{1 k}^{+}}{\partial s_{2}}-\frac{\partial E_{2 k}^{+}}{\partial s_{1}}\right), \\
& H_{3 k}^{-}=\frac{1}{\mathrm{j} \omega \mu_{k}}\left(\frac{\partial E_{1 k}^{-}}{\partial s_{2}}-\frac{\partial E_{2 k}^{-}}{\partial s_{1}}\right) .
\end{aligned}
$$

Due to continuity conditions for the tangent components of the electric and magnetic fields, $\boldsymbol{E}$ and $\boldsymbol{H}$, respectively at the boundary between the sectors $k$ and $k+1$, it can be shown that $[10,11]$

$$
\left[\begin{array}{c}
E_{k+1}^{+} \\
E_{k+1}^{-}
\end{array}\right]=\boldsymbol{M}_{k}\left[\begin{array}{c}
E_{k}^{+} \\
E_{k}^{-}
\end{array}\right],
$$

where

$$
\begin{gathered}
\mathbf{M}_{k}=\frac{1}{2}\left[\begin{array}{cc}
\left(1+\lambda_{k}\right) e^{-\alpha_{k} d_{k}} & \left(1-\lambda_{k}\right) e^{\alpha_{k} d_{k}} \\
\left(1-\lambda_{k}\right) e^{-\alpha_{k} d_{k}} & \left(1+\lambda_{k}\right) e^{\alpha_{k} d_{k}}
\end{array}\right], \\
\lambda_{k}=\frac{Z_{k+1}}{Z_{k}} .
\end{gathered}
$$

The relation between the electric field amplitude in the first layer and beyond the last one, i.e. within the area $k=n+1-$ (see Fig. 1) can be written as:

$$
\left[\begin{array}{c}
E_{1, n+1}^{+} \\
E_{1, n+1}^{-}
\end{array}\right]=\boldsymbol{P}\left[\begin{array}{c}
E_{1,1}^{+} \\
E_{1,1}^{-}
\end{array}\right],
$$

where

$$
\boldsymbol{P}=\boldsymbol{M}_{n} \boldsymbol{M}_{n-1} . . \boldsymbol{M}_{1}=\left[\begin{array}{ll}
p_{11} & p_{12} \\
p_{21} & p_{22}
\end{array}\right]
$$

is the transfer matrix for the entire skin layer. By neglecting within the $n+1$ area the wave incident onto the coordinate $s_{3}$, then with (32) and (39) it is obtained [11]:

$$
\left.\frac{E_{1,1}}{H_{2,1}}\right|_{s_{3}=0}=Z_{c}=Z_{1} \frac{p_{22}-p_{21}}{p_{22}+p_{21}} .
$$

According to the description provided above the procedure for computing $Z_{c}$ comes down to computing the $\boldsymbol{P}$ matrix, which is a simple product of $2^{\text {nd }}$ rank matrices (see formula (39)). Still, for nonlinear cases $\boldsymbol{M}_{k}$ matrix elements cannot be directly computed, as it is necessary to know 
local magnetic permeability $\mu_{k}$ values, which are dependent on the magnetic field distribution to be computed. Therefore, the concerned procedure has to be linked with another procedure (e.g. FEM, BEM, FSM - see $[8,9,11]$ ) for computing the electromagnetic field distribution within the dielectric area under the SIBC condition set at the boundary surfaces. Such a concept as well as the results of introductory numerical tests were reported elsewhere $[8,9,11]$. It consists in iterative finding electromagnetic field distribution that is self-consistent with the surface impedance distribution at the conducting sectors surfaces of the considered system. At each iteration step a linear problem is solved. That allows to compute approximately the field distribution, and based on that, to determine $Z_{c}$ distribution required for the next step of computations.

The very procedure for computing local $Z_{c}$ magnitudes is also of iterative nature. At each iteration matrices $\boldsymbol{M}_{k}$ are computed according to formula (36) for $\boldsymbol{\mu}_{k}$ values computed in the preceding iteration. Then the field distribution within the skin layer is computed from formulas (29) - (31), and in turn $\mu_{k}$ magnitudes for the next iteration are computed from $\mu=\mu(H)$ relation. The process is repeated until the magnetic field distribution within the skin layer is consistent with the magnetic permeability distribution (i.e. in each element $\Omega_{k}$ the dependence between $\mu_{k}$ and $H$ follows the magnetisation curve with the acceptable accuracy). The iteration starts with constant values for $\mu_{k}$.

It is crucial to find the values for $\mu_{k}$ in the skin layer elements. As instantaneous magnitude for absolute value of magnetic field intensity varies in time, instantaneous values for magnetic permeability can be also significantly variable, though to simplify the problem $\mu_{k}$ is treated as invariable in time. Thus, a problem arises how to set the appropriate value for $\mu_{k}$. Three approaches to determine $\mu_{k}$ seem most natural, namely

- directly from the magnetisation curve based on the magnetic field amplitude

$$
\mu_{k}=\mu\left(H_{m}\right),
$$

where $H_{m}$ corresponds to the magnetic field amplitude in the central point of the $k^{\text {th }}$ element of the skin layer,

- by averaging the $\mu=\mu(H)$ function

$$
\mu_{k}=\frac{1}{H_{m}} \int_{0}^{H_{m}} \mu(H) \mathrm{d} H
$$

- by averaging over time

$$
\mu_{k}=\frac{2}{T} \int_{0}^{T / 2} \mu\left(H_{m} \sin \omega t\right) \mathrm{d} t=\frac{1}{\pi} \int_{0}^{\pi} \mu\left(H_{m} \sin \varphi\right) \mathrm{d} \varphi .
$$

The next chapter deals with numerical experiments where all the above approaches to determine $\mu_{k}$ values were taken into consideration. 


\section{Verification of the method}

Introductory numerical test results for the provided method for determining surface impedance were presented elsewhere [11]. The procedure was found to converge effectively. After a few iterations the magnetic field distribution was found convergent with the magnetic permeability distribution within the skin layer. However, due to assumed simplification, as well as with regard to the options applicable while determining $\mu_{k}$ values, a comparison of the results to computations where distortions from the time dependencies had been considered, as well as to experimental measurements would prove essential. Such results were reported in another paper [12] where the problem of finding electromagnetic field distribution within the conductive half-space of nonlinear magnetic properties was considered. Though the magnetic hysteresis effect had not been taken into account there, the distortions from the time dependencies were considered. The finite difference method computations allowed to determine such relations as active power losses as a function of magnetic field intensity on the boundary surface. The results were also set against experimental measurements. It shall be highlighted that unit power loss can be directly related to the surface impedance with

$$
P=\frac{1}{2} \operatorname{Re}\left(Z_{c}\right) H_{0}^{2},
$$

where $H_{0}$ stands for the magnetic field strength at the boundary surface. In Fig. 2 curves denoted as 1, 2, 3 are reproduced from [12]. Discrepancies between the experimental curve no 1 and the computed one (no 3 ) were attributed to hysteresis losses not considered in the computations.

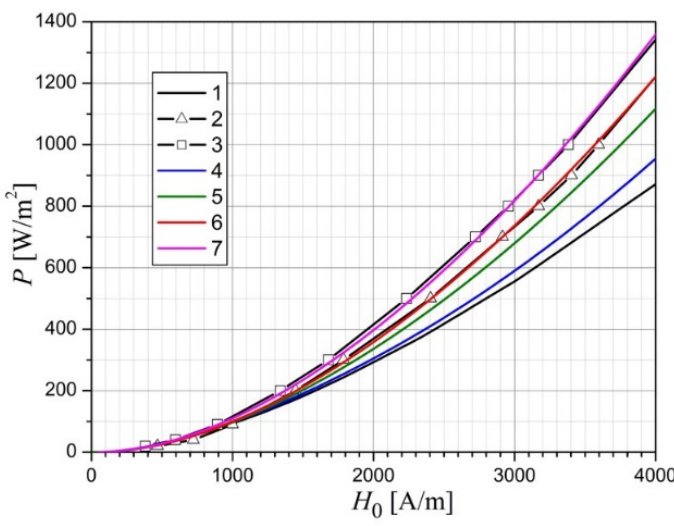

Fig. 2. Unit power losses in the conductive halfspace; 1 is a curve where constant magnetic permeability was assumed (as reported by [12]), 2 is a computation curve where time function distortion were considered (as reported by [12]), 3 is the measurement curve (as reported by [12]), $4,5,6$ are computation curves where time function distortion were disregarded and obtained for formulas (41), (42), (43), respectively, 7 is the curve for applied formula (42) with a complex magnetic permeability for $\psi=14$ degrees (compare (45))

The remaining curves in Fig. 2 represent the results obtained with the computation procedure for determining surface impedance provided hereby with the formula (44). Curves no 4, 5, and 6 represent various approaches to selecting the substitute magnetic permeability (formulas (41)-(43)). A computation curve reported in [12] was closely followed by our curve for which average magnetic permeability was applied according to the formula (42) (curve no 5). 
The hysteresis losses can be incorporated by applying a complex magnetic permeability $[13,14]$, i.e. the hysteresis curve approximated with an elliptical curve as follows:

$$
\underline{\mu}=\mu e^{j \psi} .
$$

As the paper [12] does not provide the hysteresis loop for the material for which experimental measurements had been performed, it was not possible to fully verify our method applicability to this case. Nevertheless, we managed to test compatibility of the measurement curve no 1 with our results, and prove it to be the highest for the approach where averaging magnetic permeability followed formula (42) with $\psi=14$ degrees (curve no 7).

\section{Summary}

A method for solving electromagnetic field problems by applying impedance boundary conditions is presented in the paper for systems including conducting bodies of nonlinear magnetic properties in an excited harmonic field. Nonlinear properties of the conducting areas cause the real field time functions to depart from their clear sinusoidal patterns, which increases difficulty of solving. We have proposed a method where a simplified assumption is made for the distortions from proper time functions to be disregarded. The resulting error could fall within the range acceptable for typical technical systems in electrodynamics. An obvious advantage such an assumption brings is applicability of time independent Maxwell equations for complex fields.

With the assumption made valid, three types of impedance boundary conditions were derived (see formulas (12), (21), (27)) which provided generalised SIBC in linear systems. To apply them surface impedance distribution is to be known at the conductor - dielectric boundary surfaces of the system under consideration. Therefore, a procedure for computing this quantity based on the transfer matrix is proposed. The proposed procedure has been tested by comparing computational results to computations where time function distortions were considered, and to the measurements data reported elsewhere [12].

Based on the performed comparison the following conclusions were drawn:

- Accuracy of the presented procedure is highly dependent on the option for determining substitute magnetic permeability for the skin layer elements of the system (see the differences between the curves 4, 5, and 6 in Fig. 2).

- By applying averaged magnetic permeability according to formula (42) high compatibility with the results with distortions from time dependencies considered was reached.

- By applying a complex magnetic permeability, high compatibility with the measurements was achieved.

Nevertheless, it should be noted here that scarcity of the comparative material prevents us from announcing conclusions as final. Comparison of results obtained for the provided method with detailed experimental results for various types of magnetic materials would prove particularly useful. 


\section{Appendix. Differential operations within thin-layer areas}

In this chapter formulas for basic differential operations within thin-layer areas are presented. The thin-layer area of a width of $2 \eta$ contains all the points whose distance to the specific surface $\mathrm{S}$ is either equal to $\eta$, or smaller (Fig. A1). It is assumed, that $\eta$ is significantly smaller than the curvature radius of the surface $\mathrm{S}$ at each of its points.

Then, at the surface $\mathrm{S}$ an orthogonal coordinate system $s_{1}, s_{2}$ is introduced. Any P point of the thin layer area can be defined with $s_{1}, s_{2}$ coordinates of its orthogonal projection to the surface $\mathrm{S}$ and $s_{3}$ coordinate, which is the distance from point $\mathrm{P}$ to the considered surface (see Fig. A1).

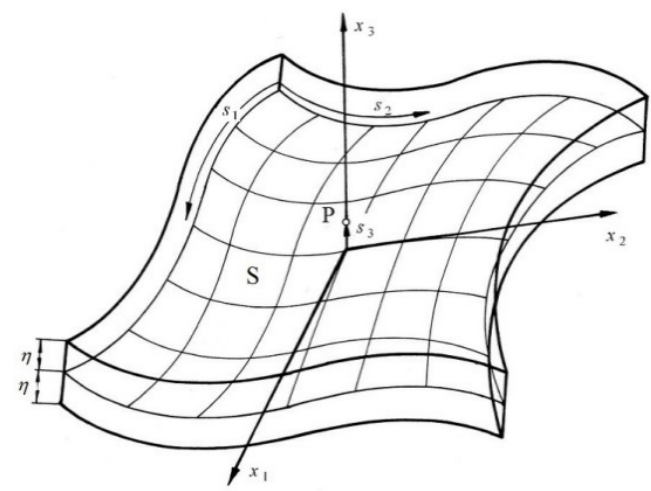

Fig. A1. A thin-layer area

Lamé coefficients for any curvilinear coordinate system $s_{1}, s_{2}, s_{3}$ can be expressed with the formula [15]

$$
h_{i}=\sqrt{\sum_{j=1}^{3}\left(\frac{\partial x_{j}}{\partial s_{i}}\right)}, \quad i=1,2,3
$$

where

$$
x_{i}=x_{i}\left(s_{1}, s_{2}, s_{3}\right)
$$

are the interrelations between coordinate $s_{i}$ and any Cartesian coordinate system $x_{1}, x_{2}, x_{3}$. The magnitudes $h_{i}$, as the lengths of certain vectors, namely the ones tangent to the appropriate coordinate lines, are not dependent on any selected Cartesian system.

To determine Lamé coefficients for the coordinate system defined above let us consider a small thin layer sector within the neighbourhood of any point $\mathrm{P}$. The Cartesian coordinate system $x_{i}$ is selected to make $x_{1}$ and $x_{2}$ axes tangent to the surface $\mathrm{S}$, and $x_{3}$ axes to cross the $\mathrm{P}$ point (see Fig. A1). It can be then safely assumed that within the concerned sector coordinates $x_{1}$ and $x_{2}$ are practically independent of the coordinate $s_{3}$, i.e. the depth at which point $\mathrm{P}$ is located, hence

$$
x_{1}=x_{1}\left(s_{1}, s_{2}\right), \quad x_{2}=x_{2}\left(s_{1}, s_{2}\right), \quad x_{3}=s_{3},
$$


which when substituted with (1) allows to arrive at

$$
h_{1}=h_{1}\left(s_{1}, s_{2}\right), \quad h_{2}=h_{2}\left(s_{1}, s_{2}\right), \quad h_{3}=s_{3} .
$$

By considering general relations determining basic differential operations for any scalar field $\varphi$ and a vector field $\boldsymbol{V}$ in the orthogonal curvilinear coordinate system [15], and by applying (A4):

$$
\begin{gathered}
\operatorname{grad} \varphi=\left[\frac{1}{h_{1}} \frac{\partial \varphi}{\partial s_{1}}, \frac{1}{h_{2}} \frac{\partial \varphi}{\partial s_{2}}, \frac{\partial \varphi}{\partial s_{3}}\right] \\
\Delta \varphi=\frac{1}{h_{1} h_{2}}\left(\frac{\partial}{\partial s_{1}}\left(\frac{h_{2}}{h_{1}} \frac{\partial \varphi}{\partial s_{1}}\right)+\frac{\partial}{\partial s_{2}}\left(\frac{h_{1}}{h_{2}} \frac{\partial \varphi}{\partial s_{2}}\right)\right)+\frac{\partial^{2} \varphi}{\partial s_{3}^{2}} \\
\operatorname{div} \boldsymbol{V}=\frac{1}{h_{1} h_{2}}\left(\frac{\partial}{\partial s_{1}}\left(h_{2} V_{1}\right)+\frac{\partial}{\partial s_{2}}\left(h_{1} V_{2}\right)\right)+\frac{\partial V_{3}}{\partial s_{3}} \\
\operatorname{rot} \boldsymbol{V}=\left[\frac{1}{h_{2}} \frac{\partial V_{3}}{\partial s_{2}}-\frac{\partial V_{2}}{\partial s_{3}}, \frac{\partial V_{1}}{\partial s_{3}}-\frac{1}{h_{1}} \frac{\partial V_{3}}{\partial s_{1}}, \frac{1}{h_{1} h_{2}}\left(\frac{\partial}{\partial s_{1}}\left(h_{2} V_{2}\right)+\frac{\partial}{\partial s_{2}}\left(h_{1} V_{1}\right)\right)\right] .
\end{gathered}
$$

As point $\mathrm{P}$ has been freely selected, the relations [A5-A8] for the coordinate system under consideration are correct within the entire thin layer sector.

The following surface differential operators are used in the paper:

- surface gradient

$$
\overline{\overline{\operatorname{grad}} \varphi} \varphi\left[\frac{1}{h_{1}} \frac{\partial \varphi}{\partial s_{1}}, \frac{1}{h_{2}} \frac{\partial \varphi}{\partial s_{2}}, 0\right]
$$

- surface Laplacian

$$
\overline{\bar{\Delta}} \varphi=\frac{1}{h_{1} h_{2}}\left(\frac{\partial}{\partial s_{1}}\left(\frac{h_{2}}{h_{1}} \frac{\partial \varphi}{\partial s_{1}}\right)+\frac{\partial}{\partial s_{2}}\left(\frac{h_{1}}{h_{2}} \frac{\partial \varphi}{\partial s_{2}}\right)\right),
$$

- surface divergence

$$
\overline{\overline{\operatorname{div}}} \boldsymbol{V}=\frac{1}{h_{1} h_{2}}\left(\frac{\partial}{\partial s_{1}}\left(h_{2} V_{1}\right)+\frac{\partial}{\partial s_{2}}\left(h_{1} V_{2}\right)\right) .
$$

\section{References}

[1] Leontovich M.A., On the approximate boundary conditions for the electromagnetic field on the surface of well conducting bodies, Investigations of Radio Waves, Academy of Sciences of USSR, Moscow (1948).

[2] Senior T.B.A., Volakis J.L., Approximate Boundary Conditions in Electromagnetics, The Institution of Electrical Engineers, London (1995).

[3] Yuferev S., Ida N., Surface Impedance Boundary Conditions. A comprehensive approach, CRC Press (2010). 
[4] Kaźmierski M., Pawłowski S., Practical application of integral equations for calculation of transformer leakage field, Archives of Electrical Engineering, vol. 45, no. 3, pp. 251-261 (1996).

[5] Lin L., Xiang C., Losses Calculation in Transformer Tie Plate Using the Finite Element Method, IEEE Transactions on Magnetics, vol. 34, no. 5 (1998).

[6] Higuchi Y., Koizumi M., Integral Equation Method with Surface Impedance Model for 3D Eddy Current Analysis in Transformers, IEEE Transactions on Magnetics, vol. 36, no. 4 (2000).

[7] Pawłowski S., Analysis of Leakage Field in Power Transformers with Use of Boundary-Iterative Method, WSEAS Transactions on Circuits and Systems, vol. 11, no. 4, pp. 1620-1627 (2005).

[8] Apanasewicz S., Pawłowski S., Plewako J., Application of an iterative method of fundamental solutions for the analysis of quasi-stationary electromagnetic field in the presence of non-linear magnetic bodies, Przeglad Elektrotechniczny (in Polish), vol. 89, no. 11, pp. 304-308 (2013).

[9] Apanasewicz S., Pawłowski S., Plewako J., Analysis for quasi-stationary electromagnetic field with ferromagnetic objects present within, Przeglad Elektrotechniczny, vol. 89, no. 12, pp. 169-173 (2013).

[10] Pawłowski S., Plewako J., The matrix method of calculating the parameters of multilayer electromagnetic screens, Przeglad Elektrotechniczny, vol. 91, no. 12, pp. 185-188 (2015).

[11] Pawłowski S., Plewako J., Analysis of nonlinear problems in electrodynamics by means of surface impedance boundary conditions (SIBC) combined with transfer matrix method, in IEEE Selected Issues of Electr. Engineering and Electronics (WZEE) (2016), DOI: 10.1109/WZEE.2016.7800192.

[12] Zakrzewski K., Determination of electromagnetic field in solid iron with application of digital method, Archives of Electrical Engineering, vol. 18, no. 3, pp. 569-585 (1969).

[13] Zakrzewski K., Berechnung der Wirk- und Blindleistung in einem ferromagnetischen Blech unter Berücksichtigung der komplexen magnetischen Permeabilität, Wiss. Z. TH Ilmenau, vol. 5, no. 16, pp. 101-105 (1970).

[14] Zakrzewski K., Kuśmierek Z., Analytic sensitiveness of power losses in electrical steels on an accuracy of determination of the elliptic hysteresis angle, Przeglad Elektrotechniczny (in Polish), vol. 2, no. 80, pp. 96-99 (2004).

[15] Trajdos T., Engineers Handbook. Mathematics (in Polish), PWN Warszawa (1986). 